Ann. Biol. anim. Bioch. Biophys., I972, 12 (I), I3-I8.

\title{
ÉVALUATION DE LA PRODUCTION DE SPERMATOZOÏDES CHEZ LE COQ
}

\author{
Michel de REVIERS \\ avec la collaboration technique de J.P. BriLlard \\ Station de Recherches avicoles, \\ Centre de Recherches de Tours, I. N. R. A., \\ 37 - Nouzilly

\section{RÉSUMÉ}

Seize coqs de même souche (M 55) et de même âge ont été séparés en un lot $\mathrm{I}$ de 7 animaux et un lot 2 de 9 animaux. Après une période prolongée d'entraînement au massage de BuRrows et QUINN (1935), la production quotidienne de spermatozoïdes a été mesurée :

- d'une part, à partir de r2 éjaculats successifs collectés soit I fois par jour (lot I), soit I fois tous les 2. jours (lot 2);

- d'autre part, à partir de numérations cellulaires (spermatides allongées et spermatozoïdes testiculaires) effectuées dans des broyats de testicules.

Le nombre de spermatozoïdes récoltés dans les éjaculats dépend de la fréquence des collectes ; ce nombre n'est représentatif de la production spermatique testiculaire que si les collectes de sperme sont effectuées I fois par jour $(r=0,9 \mathrm{I})$; cependant, cette fréquence de collecte n'a permis de récolter que $7 \circ \mathrm{p}$. roo des spermatozoïdes produits par les testicules; cette proportion n'est que de 55 p. roo dans le lot 2.

Les résultats des deux méthodes de mesure de la production spermatique n'ont donc pas la même signification; en particulier, on ne peut évaluer la production réelle de spermatozoïdes par les testicules en effectuant des collectes peu fréquentes de sperme.

\section{INTRODUCTION}

L'usage de l'insémination artificielle conduit à définir la fertilité mâle comme l'aptitude à produire la plus grande quantité possible de spermatozoildes de la meilleure qualité.

Mesurer la production de spermatozoïdes c'est donc évaluer la fertilité mâle d'un point de vue quantitatif ; mais cette production peut être déterminée soit à partir des éjaculats, soit à partir des testicules eux-mêmes. Les résultats obtenus par ces deux méthodes n'ont en général pas la même signification (cf. revue d'AMaNN, I970). 
La méthođe par éjaculations successives semble être la seule à avoir été employée pour le Coq (Burrows et Titus, I939; Swierstra et Strain, r964; Petrtjean, 1965). Aucun auteur ne paraît avoir relié les résultats obtenus par cette méthode à la production réelle des testicules. C'est ce que nous avons tenté de faire par une étude comparative dans le présent travail.

\section{MATÉRIEL E'T MÉTHODES}

Ce travail a été effectué à partir de deux lots de 7 et 9 coqs adultes de même âge (8-9 mois), de même souche (M 55), élevés dans les mêmes conditions et maintenus, pendant notre travail, dans des cages individuelles sous abri ouvert. Ces coqs ont donc été soumis aux variations climatiques locales de lumière et de température. Cette expérience s'est déroulée en octobre-novembre.

\section{I. - Obtention des éjaculats et mesures effectuées sur le sperme}

Les collectes de sperme ont été effectuées soit I fois par jour pour le lot I ( 7 coqs), soit I fois tous les 2 jours dans le lot 2 ( 9 coqs), suivant la technique de Burrows et QuinN (I937). Chaque coq a subi 25 prélèvements successifs de sperme. Les 13 premiers constituent la période préexpérimentale d'entraînement, destinée notamment à stabiliser les réserves spermatiques des voies déférentes. Les $\mathrm{r} 2$ derniers prélèvements représentent la période expérimentale proprement dite dont nous rapportons ici les résultats.

Le volume des éjaculats a été déterminé à $0,0 \mathrm{r} \mathrm{ml}$ près à l'aide d'une pipette. Leur concentration en spermatozoïdes a été déterminée dans les conditions suivantes : après homogénéisation de chaque éjaculat, une prise de $0,06 \mathrm{ml}$ (à $0,001 \mathrm{ml}$ près) a été prélevée à la micropipette et diluée dans $2 \mathrm{ml}$ d'une solution aqueuse de citrate trisodique à 3,6 p. Ioo. La densité optique de cette dilution a été mesurée à $640 \mathrm{~m} \mu$.

Pour rapporter les mesures de densité optique à la concentration en spermatozoïdes des éjaculats, la dilution précédente a été amenée à $\mathrm{r} 2 \mathrm{ml}$ par adjonction de la solution de citrate. Dans cette nouvelle dilution, les spermatozoïdes ont été comptés à l'aide d'hématimètres (Io grilles par échantillon). Enfin, les moyennes des comptages effectués pour chaque échantillon ont été rapportées aux mesures de densité optique après corrections de dilution (fig. I). Ce dernier travail a été fait pour 50 éjaculats, et a permis d'établir un abaque dont nous nous sommes servis pour les autres éjaculats. Nous avons constaté que l'influence propre du plasma séminal est négligeable dans les conditions de mesure adoptées pour notre travail.

La figure I montre qu'il existe une relation linéaire $(r: 0,93 ; \mathrm{P}<0,0 \mathrm{I})$ entre la concentration en spermatozoïdes du sperme et sa densité optique tant que celle-ci est inférieure à $\mathrm{I}$. Ceci confirme les observations d'autres auteurs (VAN TIENHOVEN et al., 1958). Au-delà decette valeur la courbe s'infléchit fortement. La concentration en spermatozoïdes ne peut alors être lue avec précision sur cette courbe ; de plus, la densité optique elle-même est alors connue avec une précision faible. Il convient donc de rediluer les échantillons de sperme qui présentent une densité optique élevée.

\section{2. - Mesures effectuées pour les testicules}

Ces mêmes cogs ont été abattus dans les 30 minutes qui ont suivi le dernier prélèvement de sperme. Les testicules ont été prélevés et pesés aussitôt après la mort des coqs. Un échantillon de 0,5 à I g, pris dans la zone équatoriale de chacun d'eux, a été pesé à I mg près, puis broyé dans du saccharose $0,25 \mathrm{M}$ pendant ${ }_{5} 5$ secondes à environ $5000 \mathrm{t} / \mathrm{mn}$ (de REviers, 197 I c).

Dix comptages hématimétriques ont été faits pour chaque échantillon de testicule, les catégories cellulaires dénombrées étant les spermatides allongées des stades $L_{6}, L_{7}$ et $L_{8}$ et les spermatozoïdes testiculaires (de REVIERS, I97I $b$ ).

A la suite d'Ortavant (1958) nous appelons :

- "coefficient d'activité testiculaire " le nombre total de spermatides allongées et de spermatozoïdes par gramme de testicule;

- " nombre testiculaire" le produit de ce coefficient par le poids testiculaire.

Connaissant le nombre testiculaire $N_{t}$ et la durée $\theta$ qui est égale au total de la durée de vie des spermatides allongées et de la durée de séjour dans les testicules des spermatozoïdes qui en 
sont issus $(\theta=4,5 j$; de Reviers non publié), nous pouvons calculer la production quotidienne $Q$ de spermatozoïdes par les testicules à l'aide de la relation

$$
Q=\frac{N_{t}}{\theta}
$$

\section{RÉSSULTATS}

\section{I. - Évaluation de la production spermatique au niveau des éjaculats}

La quantité moyenne de spermatozoïdes récoltés par éjaculat est de $\mathrm{I}, 4$ milliard dans le lot I ( $\mathrm{I}$ collecte par jour) et de I,8 milliard dans le lot 2 (I collecte tous les 2 jours). La différence entre ces résultats est significative au seuil $\mathrm{I}$ p. Ioo (test $t$ ). La quantité moyenne de spermatozoïdes récoltée par jour ou " récolte moyenne quotidienne " est donc de 0,9 milliard pour le lot 2 ; elle est très significativement inférieure à celle du lot $\mathrm{I}$.

Le nombre de spermatozoïdes récoltés dans les éjaculats présente des variations importantes d'un jour à l'autre pour l'ensemble des coqs : ces variations sont moindres dans le lot 2 que dans le lot $I$. Mais, surtout, il existe une variabilité très importante entre les coqs pour l'ensemble des collectes et cette dernière variabilité est bien plus grande que celle que nous constatons d'un jour à l'autre.

\section{2. Évaluation de la production spermatique au niveau des testicules}

Le poids testiculaire et le coefficient d'activité testiculaire ne diffèrent pas significativement d'un lot à l'autre (tabl. I). Il en résulte que les nombres testiculaires ne sont pas non plus significativement différents. Cependant ces trois critères ont des valeurs un peu moins élevées dans le lot 2 que dans le lot $\mathrm{I}$.

D'après ces résultats la production quotidienne de spermatozoïdes par les testicules est de $2, \mathrm{I}$ milliards par coq pour le lot I et de I,6 milliard pour le lot 2 .

\section{TABLEAU I}

\section{Production spermatique testiculaire chez le Coq adulte} (Automne)

Lot I : Coqs prélevés I fois par jour;

Lot 2 : Coqs prélevés $\mathrm{I}$ fois tous les 2 jours.

\begin{tabular}{|c|c|c|c|c|}
\hline & $\begin{array}{c}\text { Poids } \\
\text { testiculaire } \\
\text { total }\end{array}$ & $\begin{array}{l}\text { Coefficient } \\
\text { d'activité } \\
\text { testiculaire }\end{array}$ & $\begin{array}{l}\text { Nombre } \\
\text { testiculaire }\end{array}$ & $\begin{array}{l}\text { Production } \\
\text { spermatique } \\
\text { testiculaire }\end{array}$ \\
\hline $\begin{array}{c}\text { Lot } 1 \\
(7 \text { coqs })\end{array}$ & $20,4 \pm 2,11 \mathrm{~g}$ & $460 \cdot 10^{6}$ & $9,4 \cdot 10^{9}$ & $2,1 \cdot 10^{9}$ \\
\hline $\begin{array}{c}\text { Lot } 2 \\
(9 \text { cogs })\end{array}$ & $16,2 \pm 1,83$ & $447 \cdot 10^{6}$ & $7,1 \cdot 10^{9}$ & $1,6 \cdot 10^{\circ}$ \\
\hline Test $t$ & NS $5 \%$ & NS $5 \%$ & NS $5 \%$ & NS $5 \%$ \\
\hline
\end{tabular}


Le poids testiculaire et le coefficient d'activité testiculaire présentent chacun des variations individuelles importantes (tabl. I) ; ces deux paramètres ne sont pas liés au plan statistique. Par contre, il existe une corrélation élevée et hautement significative entre le nombre testiculaire et le poids testiculaire $(r=0,85$ pour l'ensemble des 2 lots).

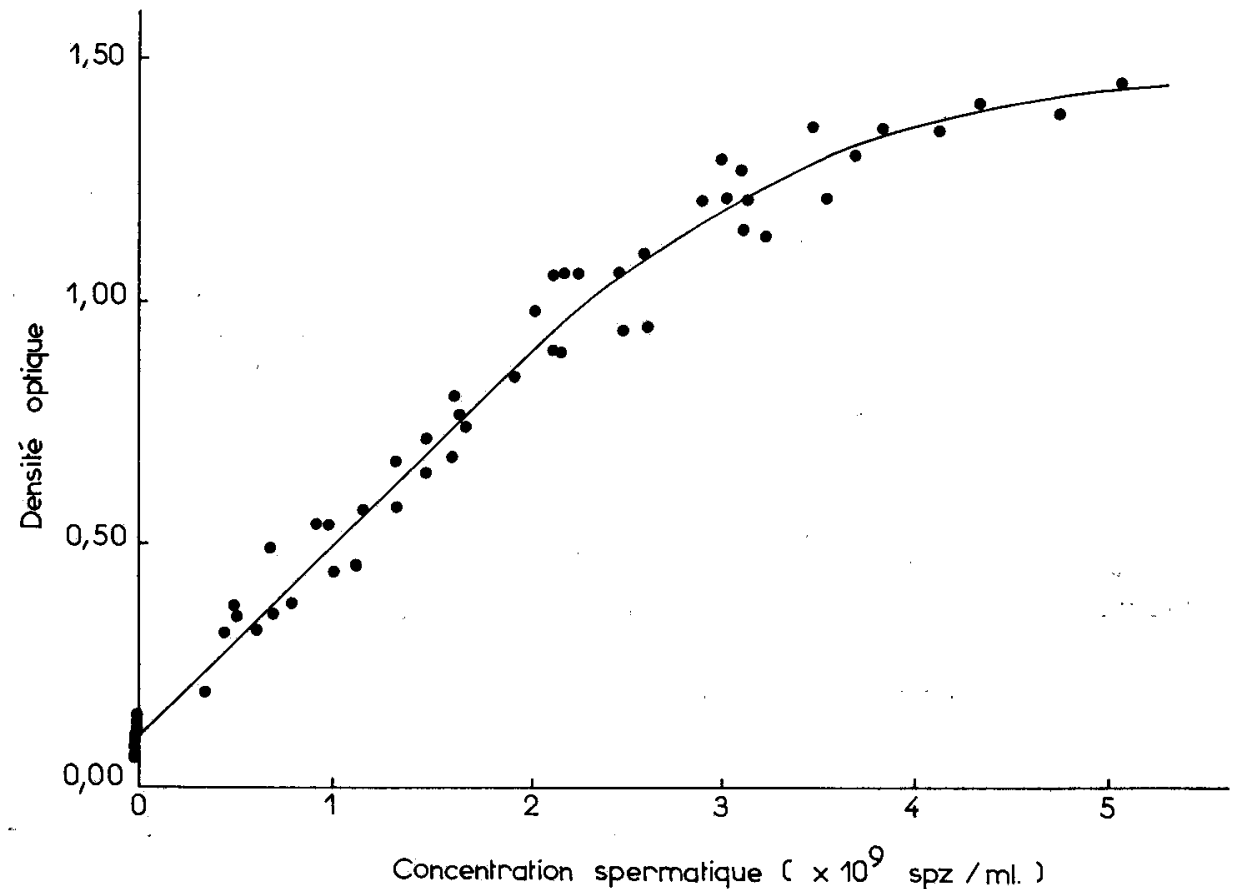

Fig. I. - Relation entre la densité optique $(\lambda=640 \mathrm{~m} \mu)$ du sperme dilué à 3 p. 100 dans une solution acqueuse de citrate trisodique a $3, p .100$ et la concentration spermatique (nombre de spermatozoides par $\mathrm{ml}$ de sperme non dilué) chez des cogs $M 55$ à l'automne.

3. - Relations entre les résultats des deux méthodes de mesure de la production de spermatozoïdes

Même en effectuant des collectes quotidiennes de sperme, nous n'avons récolté

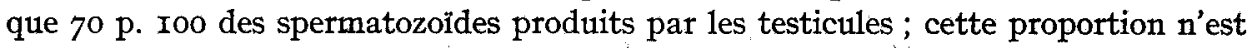
que de 55 p. Ioo lorsque les collectes sont effectuées $I$ fois tous les 2 jours.

De plus, nous n'observons de corrélation entre la "récolte moyenne quotidienne" de spermatozoïdes et la production testiculaire que pour le lot I (prélevé $\mathrm{I}$ fois par jour) ; cette corrélation est de $0,9 \mathrm{I}(\mathrm{P}<\mathrm{o}, \mathrm{OI})$; dans le lot 2 elle est de $-0,2 \mathrm{I}$ et n'est pas significative au seuil 5 p. roo.

\section{DISCUSSION}

En montrant que chez le Coq la " récolte moyenne quotidienne " dépend de la fréquence de collecte, nos résultats confirment des observations faites notamment par Swierstra et Strain (I944) et Petitijean (I965). 
Mais notre étude apporte des faits nouveaux importants en montrant :

- ou bien que les résultats des 2 méthodes de mesure de la production spermatique sont en corrélation élevée (lot I), mais la quantité de spermatozoìdes récoltée n'est alors que de 70 p. Ioo de la production testiculaire des coqs ;

- ou bien qu'il n'y a pas de corrélation significative entre les résultats de deux méthodes (lot 2).

Ces deux mesures de la production de spermatozoïdes n'ont donc pas la même signification chez le Coq.

Une fraction importante de la production testiculaire des coqs n'apparait pas dans leurs éjaculats ( $30 \mathrm{p}$. Ioo dans le lot I et $45 \mathrm{p}$. Ioo dans le lot 2 ). Trois possibilités nous paraissent pouvoir expliquer ce phénomène, soit que les coqs éjaculent spontanément entre deux collectes successives, soit qu'ils émettent des spermatozoïdes avec l'urine et les fèces d'une manière plus ou moins continue, soit encore que des spermatozoïdes soient résorbés au cours de leur transit dans les voies déférentes. L'importance relative de ces processus n'a pu pour le moment être chiffrée chez le Coq. Il faut noter que, si leur résultante aboutit à la perte d'un nombre élevé de spermatozoïdes chez des coqs dont le sperme est prélevé régulièrement, cette perte est encore bien plus grande chez des coqs non prélevés qui n'accumulent en moyenne que 7 à 8 jours de production spermatique dans leurs voies déférentes (de REVIERS, I97I c).

Pour le lot 2 (coqs prélevés I fois tous les 2 jours), nous constatons non seulement que la " récolte moyenne quotidienne " n'est pas liée à la production spermatique des testicules, mais encore que les variations observées d'un éjaculat à l'autre sont moins importantes que dans le lot $I$. On peut penser qu'un mécanisme régulateur est intervenu chez les coqs du lot 2 en agissant sur le nombre de spermatozoïdes collectés dans les éjaculats. Nous constatons en effet que les réserves spermatiques des voies déférentes représentent environ 4,5 éjaculats pour le lot 2 mais seulement 3 éjaculats pour le lot I. Il est donc possible que les réserves spermatiques des voies déférentes du lot 2 aient été maintenues à un niveau suffisant pour avoir ce rôle régulateur.

Du fait que la quantité de spermatozoïdes récoltés dans des éjaculats au cours de collectes peu fréquentes ne représente pas la production spermatique réelle des coqs, nous sommes conduit à remettre en question la valeur de la plupart des publications ayant trait à l'influence de tel ou tel facteur sur la spermatogenèse du Coq, puisque cette influence n'a été le plus souvent appréciée que par des collectes de sperme effectuées une fois par semaine, voire même une fois par mois.

Il nous semble que la meilleure évaluation de la production spermatique réelle consiste à mesurer le nombre testiculaire. Cependant cette méthode présente deux inconvénients majeurs :

- elle nécessite une connaissance très précise de la durée de vie des spermatides allongées et de la durée de séjour des spermatozoïdes dans les testicules ;

- elle ne permet d'évaluer la production spermatique d'un coq que pour un instant donné ; l'évolution individuelle de cette production ne peut donc être suivie dans le temps.

Ces inconvénients se trouvent palliés dans la méthode par éjaculations successives mais les résultats de cette dernière ne sont en relation avec la production spermatique 
réelle des testicules que si la fréquence des collectes est suffisamment élevée (ici I fois par jour) ; il est vraisemblable que cette fréquence doit être adaptée en fonction du niveau atteint par la production testiculaire.

Reçu pour publication en mai 1971.

\section{SUMMARY}

\section{ESTIMATION OF SEMEN PRODUCTION IN THE MALE FOWL}

Sixteen 8 months old M. 55 cockerels were distributed into two batches ( 7 vs. 9 birds) and submitted to a long training to Burrows and Quinn's massage (1935). Their daily sperm output (DSO) was estimated from $\mathrm{r} 2$ consecutive ejaculations collected daily (batch $\mathrm{r}$ ) or every other day (batch 2). The daily sperm production (DSP) was estimated by counting elongated spermatids and spermatozoa in testicular homogenates.

The DSO varied depending on the frequency of semen collection (1.35 $\times 10^{9} \mathrm{spz}$. in batch I vs. $0.91 \times 10^{9} \mathrm{spz}$. in batch 2 ), which is in accordance with the literature.

There was a highly significant correlation coefficient $(r=0.9 \mathrm{I})$ between DSO and DSP in batch $\mathrm{I}$. There was no significant correlation in batch 2, possibly because of the presence of stored semen in the vasa deferentia.

Only 70 per cent of the DSP was collected in the daily ejaculated birds, vs. 55 per cent only in batch 2 birds. Thus, a considerable loss of spermatozoa occurs whose mechanism is still unknown in the species.

\section{RÉFÉRENCES BIBLIOGRAPHIQUES}

Amann R. P., 197o. Sperm production rates; in : The Testis, Johnson A. D., Gomes W. R., VAN Demark N. L., 1, 433-482.

Burrows W. H., Quins J. P., 1935. A method of obtaining spermatozoa from the domestic fowl. Poult. Sci., 14, $25 \mathrm{I}-254$.

Burrows W. H., Titus H. W., I939. Some observations on the semen production of the male fowl. Poult. Sci., 18, 8-10.

Ortavant R., 1968. Le cycle spermatogénétique chez le Belier. Thèse Doctorat ès Sciences, Paris, I $27 \mathrm{p}$.

Petitjean M., r965. Recherches sur l'estimation du pouvoir fécondant des cogs. Thèse Ingénieur C. N. A. M., 79 p.

Reviers M. de, I968. Détermination de la durée des processus spermatogénétiques chez le Coq à l'aide de Thymidine tritiée. VI $I^{\mathrm{e}}$ Cong. intern. Reprod. anim. Insém. artif., Paris, 1, I83-I84.

Reviers M. de, I97I $b$. Le développement testiculaire chez le Coq. II. Morphologie de l'épithélium séminifère et établissement de la spermatogenèse. Ann. Biol. anim. Biophys. Bioch. (sous presse).

REviers M. de, I971 c. La production spermatique du Coq. I. Estimation des réserves spermatiques dans les voies déférentes. Ann. Biol. anim. Biophys. Bioch. (sous presse).

Swterstra E. E., Strain J. H., rg64. Sperm output of White Leghorn roosters ejaculated daily or every other day for eight weeks. Poult. Sci., 43, I368.

Tienhoven A. Van, Steel R. G. D., Duchaine S. A., r958. Diluents for turkey semen. Poult. Sci., 37, 47-53. 